LA-UR-01-3969

Approved for public release; distribution is unlimited.

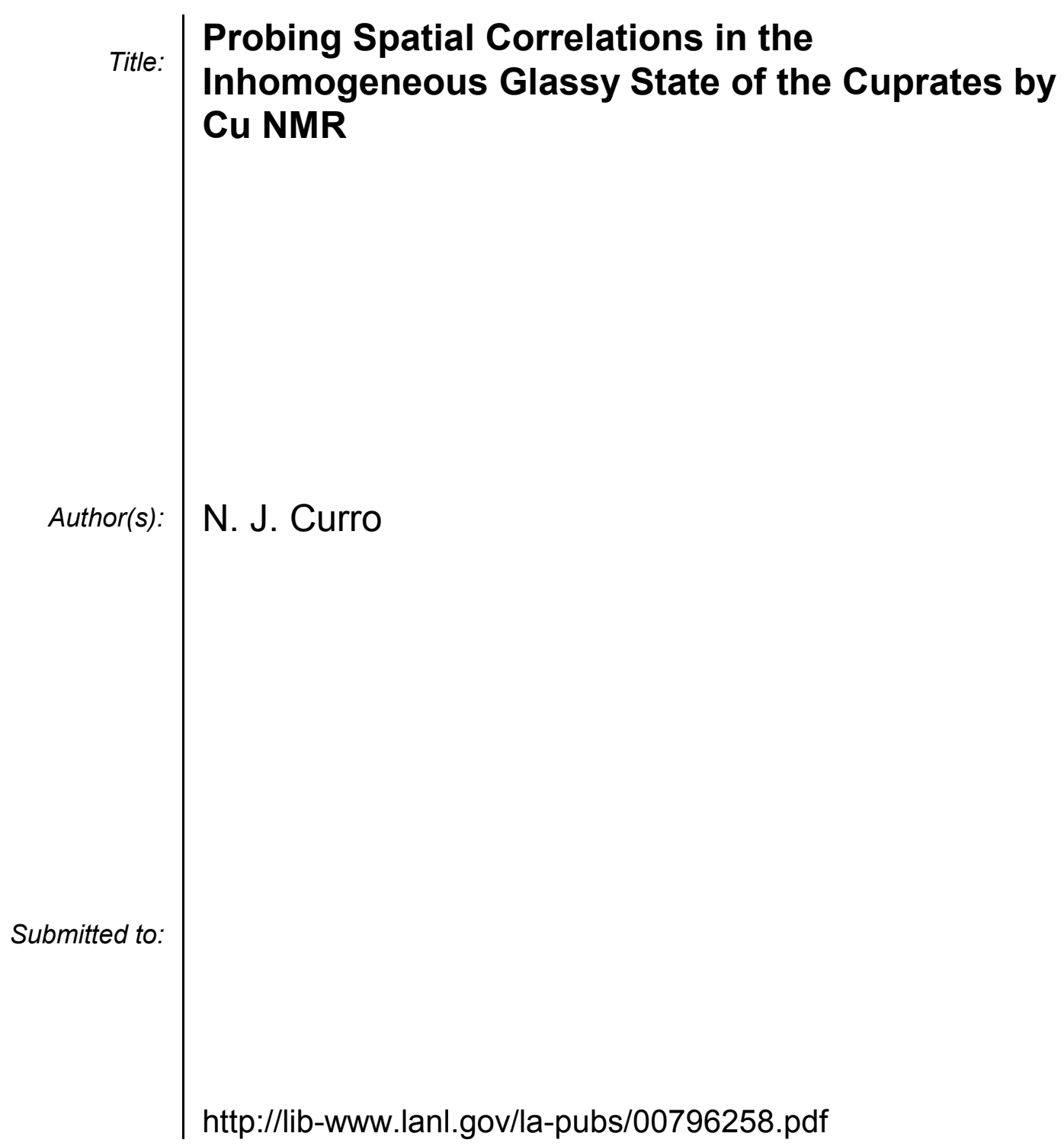

Los Alamos National Laboratory, an affirmative action/equal opportunity employer, is operated by the University of California for the U.S. Department of Energy under contract W-7405-ENG-36. By acceptance of this article, the publisher recognizes that the U.S. Government retains a nonexclusive, royaltyfree license to publish or reproduce the published form of this contribution, or to allow others to do so, for U.S. Government purposes. Los Alamos National Laboratory requests that the publisher identify this article as work performed under the auspices of the U.S. Department of Energy. Los Alamos National Laboratory strongly supports academic freedom and a researcher's right to publish; as an institution, however, the Laboratory does not endorse the viewpoint of a publication or guarantee its technical correctness. 


\title{
Probing Spatial Correlations in the Inhomogeneous Glassy State of the Cuprates by $\mathrm{Cu}$ NMR
}

\author{
N. J. Curro \\ Condensed Matter and Thermal Physics, Los Alamos National Laboratory, Los Alamos, NM 87545, USA
}

\begin{abstract}
We discuss the crossover of the form of the $\mathrm{Cu}$ NMR spin echo decay at the onset of $\mathrm{Cu}$ wipeout in lanthanum cuprates. Experimentally, the echo decay undergoes a crossover from Gaussian to exponential form below the temperature where the $\mathrm{Cu}$ NMR intensity drops. The wipeout and the change in behavior both arise because the nuclei experience spatially inhomogeneous spin fluctuations at low temperatures. We argue that regions where the spin fluctuations remain fast are localized on length scales of order 1-2 lattice spacings. The inhomogeneity is characterized by the local activation energy $E_{a}(\mathbf{r})$; we estimate the functional form of $E_{a}(\mathbf{r})$ for points where $E_{a}(\mathbf{r}) \sim 0$.
\end{abstract}

The lanthanum cuprate high temperature superconductors have received a considerable amount of attention in recent years because elastic neutron scattering experiments in rare-earth co-doped $\mathrm{La}_{1.8-x} \mathrm{Eu}_{0.2} \mathrm{Sr}_{x} \mathrm{CuO}_{4}$ and $\mathrm{La}_{1.6-x} \mathrm{Nd}_{0.4} \mathrm{Sr}_{x} \mathrm{CuO}_{4}$ have revealed ordering of doped holes into charged stripes that constitute anti-phase domain walls producing incommensurate antiferromagnetic (AF) order in the intervening undoped domains [1]. Since the initial discovery, several studies have revealed that the order is more complex than initially thought; instead of exhibiting a well-defined thermodynamic transition temperature the spin system appears to exhibit glassy behavior. [2,3] Nuclear Magnetic Resonance (NMR) and Nuclear Quadrupolar Resonance (NQR) studies have shown that this glassy behavior is accompanied by inhomogeneous slowing of the local spin fluctuations, and provide a direct measure of the distribution of activation energies giving rise to the inhomogeneous distribution. [4] This distribution can be characterized by measuring the temperature dependence of the La $T_{1}$; surprisingly this distribution is remarkably independent of doping (at least up to optimally doped), local structure, and doping location in the unit cell. Recent theoretical work suggests that the ubiquity of this glassy behavior may be intrinsic to doped hole systems. [5] The wipeout phenomenon of the $\mathrm{Cu}$ NMR spin echo intensity also arises from the development of these inhomogeneous spin fluctuations, with a broad distribution of fluctuation times. In fact, the temperature dependence of the $\mathrm{Cu}$ wipeout can be entirely explained by the distribution of activation energies measured by the La $T_{1}$.

However, measurements of the $\mathrm{La}$ and $\mathrm{Cu} T_{1}$ or the $\mathrm{Cu}$ wipeout reveal information only about the local spin fluctuations at the nucleus under observation. This allows one to determine the distribution $\mathcal{P}\left(E_{a}\right)$ of activation energies $E_{a}$ characterizing the glassy behavior. The echo decay of the $\mathrm{Cu}$, on the other hand, reveals information about not only the particular $\mathrm{Cu}$ nucleus under observation, but its surrounding neighbors as well. This serendipitous fact allows one to make conclusions about $E_{a}(\mathbf{r})$, the activation energy at position $\mathbf{r}$. In fact, the form of the echo decay of the $\mathrm{Cu}$ provides two important pieces of information: (i) the sites $\mathbf{r}_{i}$ in the crystal such
$E_{a}\left(\mathbf{r}_{i}\right) \sim 0$ are separated by a distance of at least several unit cell lengths, $a$, and (ii) the effective radius of the minima around $\mathbf{r}_{i}$ is approximately $1-2 a$.

In general the decay of the spin echo intensity (the echo decay) of a nucleus is a complex, many body problem involving couplings between many neighbors. In the cuprates the echo decay of a nucleus located at the origin is determined by the Hamiltonian $\mathcal{H}=$ $\sum_{\mathbf{r}} a_{x}(\mathbf{r}) I_{x}(0) I_{x}(\mathbf{r})+a_{y}(\mathbf{r}) I_{y}(0) I_{y}(\mathbf{r})+a_{z}(\mathbf{r}) I_{z}(0) I_{z}(\mathbf{r})$, where the sum is over all nuclear sites. The couplings $a_{\alpha}(\mathbf{r})$ are given by dipolar couplings, indirect couplings involving an RKKY type mechanism, or both. [6] For arbitrary $a_{\alpha}(\mathbf{r})$ this problem has not been solved, although significant theoretical advances have been made recently. [7] Fortunately, it turns out that the nuclear couplings in the planes of the cuprates are dominated by the indirect channel, wherein lies the utility of $\mathrm{Cu}$ NMR to determine spatial properties such as the antiferromagnetic correlation length, $\xi$. [8] For example, studies invoking the strong $\mathrm{O}-\mathrm{Cu}$ coupling in the plane have lead to important conclusions about the effects of impurities, as well as put constraints on theories of the dynamical susceptibility, $\chi^{\prime}(\mathbf{q})$. $[9,10]$ For the $\mathrm{Cu}-\mathrm{Cu}$ couplings $a_{z}(\mathbf{r}) \gg$ $a_{x}(\mathbf{r}), a_{y}(\mathbf{r})$. In this limiting case $I_{z}(0)$ and $\mathcal{H}$ commute, allowing an exact determination of the echo decay of the nucleus at $\mathbf{r}=0: M(t)=M_{0} \exp \left[-t^{2} \sum_{\mathbf{r}} a_{z}^{2}(\mathbf{r}) / 16\right]$, where the sum is over like neighbors. [8] A like nucleus is one that resonates at the same frequency as the one under observation; an unlike nucleus resonates at a different frequency. Typically, the echo decay is written in terms of a Gaussian time decay constant:

$$
T_{2 G}^{-2}=\frac{1}{8} \sum_{\mathbf{r}} a_{z}^{2}(\mathbf{r}) .
$$

$T_{2 G}$ gives information about the RKKY interactions giving rise to the indirect $\mathrm{Cu}-\mathrm{Cu}$ coupling. [8] In fact, Pennington, and later Thelen showed that $a_{z}(\mathbf{r})$ can be written in terms of the real part of the dynamic susceptibility, $\chi^{\prime}(\mathbf{q})$; using the MMP form for $\chi^{\prime}(\mathbf{q})$ one finds $T_{2 G} \sim 1 / \xi$, where $\xi$ is the antiferromagnetic correlation length. $[8,11]$

Studies of $T_{2 G}$ in the cuprates usually invoke the assumption that the couplings are time independent over 


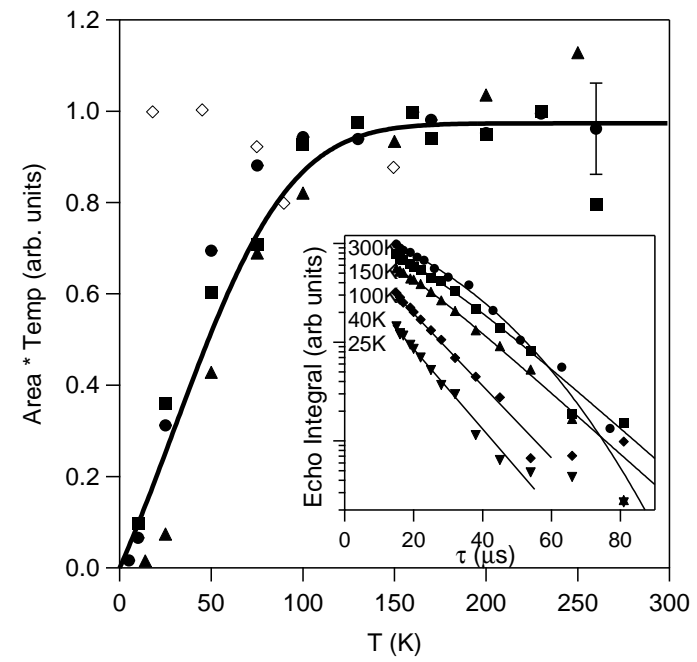

FIG. 1. NMR measurements in $\mathrm{La}_{1.65} \mathrm{Eu}_{0.2} \mathrm{Sr}_{0.15} \mathrm{CuO}_{4}$ showing the wipeout of $\mathrm{Cu}$ signal. The solid symbols represent $\mathrm{Cu}$ data: (squares) $77 \mathrm{MHz}, H_{0} \perp c$; (circles) $86 \mathrm{MHz}$, $H_{0} \perp c$; (triangles) $95 \mathrm{MHz}, H_{0} \| c$. The open diamonds represent La data for $H_{0} \| c$ at $35.9 \mathrm{MHz}$. The solid line is a plot of the calculated intensity as described in the text. INSET: $H_{0} \| c{ }^{63} \mathrm{Cu}$ echo size versus the pulse spacing $\tau$ is plotted for a series of temperatures.

the time scale of the echo decay, or equivalently, $T_{1} \gg$ $T_{2 G}$. In most cases this static assumption is valid. However, in cases where $T_{1}$ exhibits inhomogeneous glassy behavior (i.e., below the onset of $\mathrm{Cu}$ wipeout) this assumption is not necessarily valid; in fact, for wipeout to occur, $T_{1} \leq T_{2 G}$ locally. Therefore it is necessary to understand how the echo decay is modified when the static assumption is relaxed. This problem was studied successively by Walstedt and Cheong, [12], Recchia and Pennington, [13], and Curro and Slichter. [14] It turns out that one can write the Gaussian part of the echo decay of the nucleus at $\mathbf{R}$ as

$$
M_{G}(\mathbf{R}, t)=\exp \left[\frac{-t^{2}}{16} \sum_{\mathbf{r}} a_{z}^{2}(\mathbf{r}-\mathbf{R}) f\left(\frac{t}{T_{1}(\mathbf{r}-\mathbf{R})}\right)\right],
$$

where $f(x)=\phi_{L}(x)+c_{1} \phi_{U}\left(c_{2} x\right)$. Here the constant $c_{1}=$ $\left({ }^{65} \mathcal{P}^{65} \gamma^{2}\right)\left({ }^{63} \mathcal{P}^{63} \gamma^{2}\right)=0.516$, accounts for the isotopic distribution of $\mathrm{Cu}$ which gives rise to both like and unlike nuclear couplings, $c_{2}=\left({ }^{65} \gamma /{ }^{63} \gamma\right)^{2}$,

$$
\phi_{U}(x)=x^{-2}\left(x+4 e^{-x / 2}-e^{-x}-3\right)
$$

for unlike nuclei and

$$
\phi_{L}(x)=8 x^{-2}\left(5 x / 2+9 e^{-x / 2}-2 e^{-x}-7\right)
$$

for like nuclei. If $T_{1}$ is spatially uniform then $f\left(t / T_{1}(\mathbf{r}-\mathbf{R})\right)$ can be taken out of the summation, and Eq. (2) can be written trivially as $M_{G}(t)=$ $\exp \left[-t^{2} f\left(t / T_{1}\right) / 2 T_{2 G}^{2}\right]$. In homogeneous systems such as
$\mathrm{YBa}_{2} \mathrm{Cu}_{4} \mathrm{O}_{8}$, the temperature dependence of $T_{1}$ typically leads to minor corrections to the Gaussian form of the $\mathrm{Cu}$ echo decay. [15]

In cuprate systems that exhibit inhomogeneous spin fluctuations the $\mathrm{Cu}$ echo decay can be modified significantly. Most notably, the echo decay exhibits a dramatic, sharp drop on times scales shorter than what can typically be measured by an NMR spectrometer, an effect known as wipeout, in which the number of observable nuclei effectively is reduced. The full expression for the echo decay is given by:

$$
M(t)=\sum_{\mathbf{R}} M_{G}(\mathbf{R}, t) \exp \left(\frac{-t}{T_{2 R}(\mathbf{R})}\right)
$$

where $M_{G}(\mathbf{R}, t)$ is given by Eq. (1), $T_{2 R}^{-1}(\mathbf{r})=(\beta+$ $R) T_{1}^{-1}(\mathbf{r})$ is the Redfield term and $\beta$ and $R$ are constants. [6] The Redfield term accounts for on-site spin lattice relaxation over the time scale of the echo decay. $T_{1}(\mathbf{r})$ is given by the local activation energy:

$$
T_{1}^{-1}(\mathbf{r})=\gamma^{2} h_{\mathrm{hf}}^{2} \tau_{\infty} \exp \left(E_{a}(\mathbf{r}) / k_{B} T\right),
$$

where $\gamma$ is the gyromagnetic ratio, $h_{\mathrm{hf}}$ is the hyperfine field at the nucleus, $\tau_{\infty}$ is the spin fluctuation time at high temperatures, and $T$ is the temperature. The spin echo signal that is left over, then, arises from $\mathrm{Cu}$ nuclei that remain in regions of longer $T_{1}$, or equivalently small $E_{a}$. The activation energies $E_{a}$ are distributed with distribution function $\mathcal{P}\left(E_{a}\right)$, as in Ref. [4]. The Redfield term leads to wipeout since at low temperatures a significant fraction of nuclei experience $T_{1}$ 's that are too fast to detect with the spectrometer. One might inquire what interactions these remaining nuclei experience: are they coupled to nuclei that experience similar $T_{1}$ 's, or are they coupled to nuclei with much faster $T_{1}$ 's? We argue that the latter case predominates, and is the reason for the change in the character of the echo decay. Experimentally it is found that the echo decay crosses over from Gaussian to exponential character. [4,16,17] (see Fig. (1)) We have argued previously that this crossover reflects regions of small spatial extent with fast spin fluctuations; here we present a more quantitative description of the phenomenon.

Consider a hypothetical spatial dependence of activation energies $E_{a}(\mathbf{r})$ such as that shown in Fig. (2). Note that at the observable nuclei are those near the minimum in the figure. It is useful to consider how the spatial dependence of $T_{1}(\mathbf{r})$ affects the echo decay of the remaining observable nuclei when the system becomes inhomogeneous. To address this question we analyze Eq. (5) with a specific model for the dynamic susceptibility. In order to calculate the indirect couplings, we adopt the expression used by Haase, Morr and Slichter et al, for the real space $\chi_{\mathrm{M} M P}:[18,19]$

$$
\chi^{\prime}(\mathbf{r}>\mathbf{0})=\frac{\alpha}{4 \pi} \cos \left(\frac{\pi x}{a}\right) \cos \left(\frac{\pi y}{a}\right) K_{0}\left(\frac{r}{\xi}\right),
$$




$$
\chi^{\prime}(\mathbf{r}=\mathbf{0})=\frac{\alpha}{4 \pi} \ln \left(1+4 \pi \xi^{2}\right),
$$

where $\alpha$ is a temperature independent constant, $\xi$ is the antiferromagnetic correlation length, and $K_{0}(x)$ is the zeroth order modified Bessel function of the second kind.

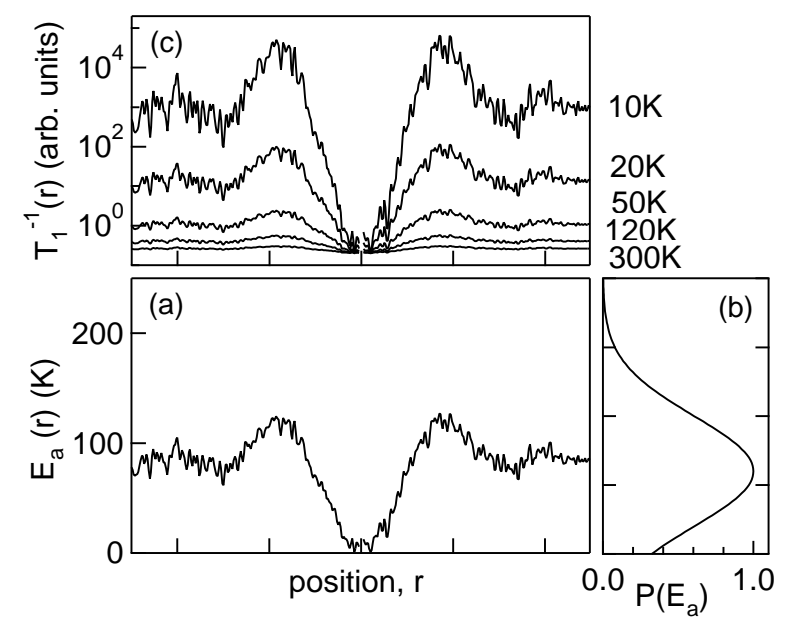

FIG. 2. (a) A hypothetical spatial distribution $E_{a}(\mathbf{r})$ versus $r$ in the vicinity of a minimum in $E_{a}$. (b) The distribution $\mathcal{P}\left(E_{a}\right)$, with parameters typical for $\mathrm{La}_{1.65} \mathrm{Eu}_{0.2} \mathrm{Sr}_{0.15} \mathrm{CuO}_{4}$. (c) The resulting spatial distribution of $T_{1}$ 's at a series of temperatures. Note that $T_{1}$ 's get more spatially inhomogeneous as the temperature is lowered.

Note that this expression assumes translation invariance; however this assumption is not necessarily valid in the case of inhomogeneous spin fluctuations. Nevertheless this expression has been successful in describing the dynamical susceptibility in homogeneous cuprates as measured by neutron scattering and NMR. [20] Given a specific form for $\chi^{\prime}(\mathbf{r})$ the indirect couplings $a_{z}(\mathbf{r})$ are given by: [18]

$$
\begin{array}{r}
\left(g \mu_{B}\right)^{2} a_{z}(\mathbf{r})=A_{z}^{2} \chi^{\prime}(\mathbf{r})+2 A_{z} B \sum_{m=1}^{4} \chi^{\prime}\left(\mathbf{r}-\mathbf{b}_{m}\right) \\
+B^{2} \sum_{m, n=1}^{4} \chi^{\prime}\left(\mathbf{r}-\mathbf{b}_{n}-\mathbf{b}_{m}\right)
\end{array}
$$

where $\mathbf{b}_{m}$ are the four nearest neighbor $\mathrm{Cu}$ sites, $A_{z}=$ $1.528 \mu \mathrm{eV}$ is the on-site hyperfine coupling and $B=-A / 4$ is the transferred hyperfine coupling to the nearest neighbor $\mathrm{Cu}$ sites.

In order to estimate an appropriate form for the spatial dependence of $T_{1}$ in the vicinity of a minimum, we first consider the distribution function $\mathcal{P}\left(E_{a}\right)$ for $E_{a}$ close to zero. In fact, given $E_{a}(\mathbf{r}), \mathcal{P}\left(E_{a}\right)$ is given by:

$$
\mathcal{P}\left(E_{a}\right)=1 /\left|\nabla E_{a}(\mathbf{r})\right|=\left|r\left(\frac{d E_{a}}{d r}\right)^{-1}\right|,
$$

so for $r \sim r_{i}$ we have $E_{a}(r)=\left(r-r_{i}\right)^{2} / 2 \mathcal{P}_{0} \lambda^{2}$, where $\mathcal{P}_{0}=\mathcal{P}\left(E_{a}=0\right)$, and $\lambda$ is a length scale that characterizes the size of the regions in space where $E_{a}$ is close to zero. If $\mathcal{P}_{0}=0$, we have $E_{a}(r)=\left(\frac{d \mathcal{P}_{0}}{d E_{a}}\right)^{-1 / 2}\left(\left(r-r_{i}\right) / \lambda\right)$, where $d \mathcal{P}_{0} / d E_{a}=d \mathcal{P}\left(E_{a}=0\right) / d E_{a}$. Thus we use two test cases for the spatial dependence of $T_{1}$, defining $W(\mathbf{r})=1 / T_{1}(\mathbf{r})$ :

$$
\begin{array}{rlr}
W_{e}(\mathbf{r}) & =W_{0} \exp \left(\left(r-r_{i}\right) / l\right) & \text { caseI } \\
W_{G}(\mathbf{r}) & =W_{0} \exp \left(\left(r-r_{i}\right)^{2} / 2 l^{2}\right) \quad \text { caseII, }
\end{array}
$$

where $l=k_{B} T \sqrt{d \mathcal{P}_{0} / d E_{a}} \lambda$ for case I, $l=\sqrt{\mathcal{P}_{0} k_{B} T} \lambda$ for case II, and $W_{0}=\gamma^{2} h_{\mathrm{hf}}^{2} \tau_{\infty}$. We then calculate $M(t)$ (Eq. (5)) numerically on an $N \times N$ lattice with $N=11$. The results are shown in Figs. (3) and (4). Note that $T_{2 G}$ is defined as in Eq. (1). Here we use $\xi=2 a$ and $\alpha=$ 1.0. Although it is not possible to determine the absolute value of $\xi$, it is not unreasonable to expect $\xi \sim 2-3 a$, at least in regions of fast spin fluctuations (slow $T_{1}$ ).

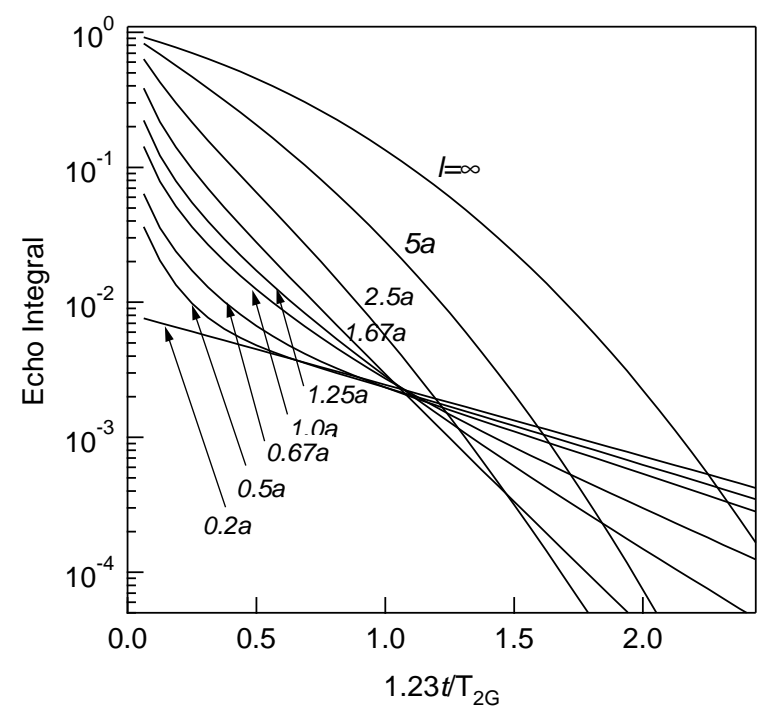

FIG. 3. Case I: The calculated $M(t)$ versus $t$ for $\xi=2 a$, with $W(\mathbf{r})=W_{e}(\mathbf{r})$ and $W_{0}=0.25 / T_{2 G}$.

In both cases, as the degree of inhomogeneity grows (as $l$ shortens, or as $T$ decreases), there are two important changes in the form of the echo decay. First, the echo decays drastically at short times due to the fast onsite relaxation from the Redfield term $\exp \left(-t / T_{2 R}(\mathbf{R})\right)$. This is the $\mathrm{Cu}$ wipeout. Secondly, the echo decay from the remaining sites gradually becomes more exponential, as seen experimentally (see Fig. (1)). This crossover occurs when $l \leq \xi$. In other words, when the neighbors that have a significant coupling (those within a radius $\xi$ ) begin to undergo fast spin lattice fluctuations, they behave as unlike nuclei; essentially they behave as strongly coupled independent fluctuating moments. As the fluctuation rate of these neighbors gets faster, the echo decay rate becomes exponential, and eventually the fast fluctuations of the neighbors decouples from the nuclei under 
observation. In the extreme limit, the nucleus at $\mathbf{r}=\mathbf{0}$ is the only one with $T_{1}$ sufficiently slow to be observable, and all of the neighbors are fluctuating so fast they do not contribute to the echo decay; thus the single nucleus at the origin decays solely due to the local Redfield term.

Note that for case II the spin lattice relaxation rate changes less drastically between the origin and the nearest neighbor sites. As a result, the echo decay, which is dominated by the coupling to the nearest neighbor sites, is not as strongly affected by the onset of the inhomogeneity $\left(T_{1}(\mathbf{r})\right.$ is locally more homogeneous). As seen in Figs. (3) and (4), $W_{G}(\mathbf{r})$ results in an echo decay that has a slightly more positive curvature at short times than $W_{e}(\mathbf{r})$. Although one cannot distinguish between the two cases experimentally, qualitatively the echo decay curves in Fig. (1) seem to match more those in case I. In other words, for $T=25 \mathrm{~K}$, the echo decay in Fig. (1) looks like the echo decay in Fig. (3) for $l \approx 1-2 a$.

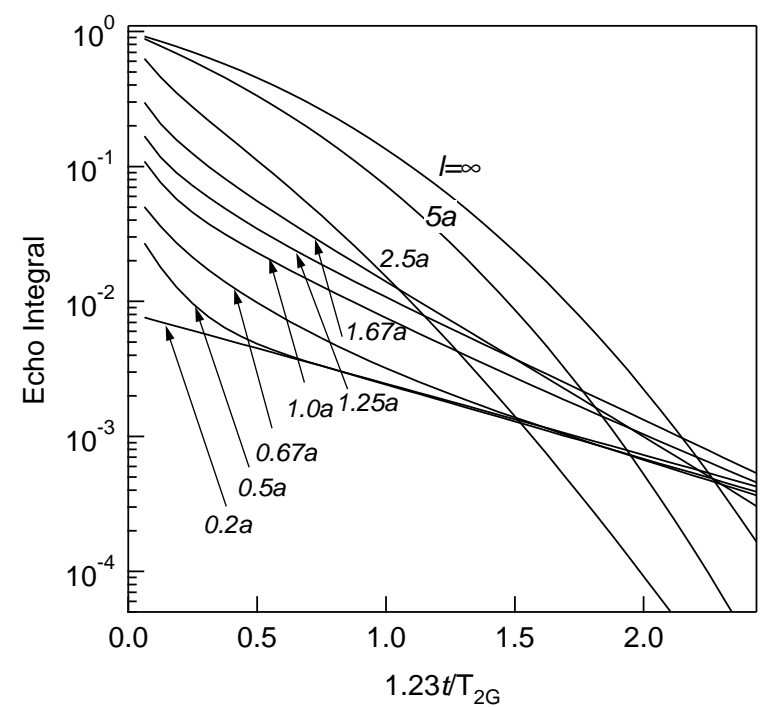

FIG. 4. Case II: The calculated $M(t)$ versus $t$ for $\xi=2 a$, with $W(\mathbf{r})=W_{G}(\mathbf{r})$ and $W_{0}=0.25 / T_{2 G}$.

We can now estimate $\lambda$, the effective radius of the regions in space with low activation energy. To do so, we take advantage of the fact that the wipeout fraction, $I_{0}(T)$ is given by:

$$
I_{0}(T)=\int_{0}^{\kappa k_{B} T} \mathcal{P}\left(E_{a}\right) d E_{a},
$$

where $\kappa \approx 1.5$ is defined as in [4]. Therefore, we have:

$$
\begin{aligned}
\mathcal{P}_{0} & =\frac{1}{\kappa k_{B}}\left(\frac{d I_{0}}{d T}\right)_{T=0} \\
\frac{d \mathcal{P}_{0}}{d E_{a}} & =\frac{1}{\left(\kappa k_{B}\right)^{2}}\left(\frac{d^{2} I_{0}}{d T^{2}}\right)_{T=0},
\end{aligned}
$$

where $I_{0}(T)$ is measured experimentally, and is shown if Fig. (1). From the data $\mathcal{P}_{0} \approx 7 \times 10^{-3} K^{-1}, d \mathcal{P}_{0} / d E_{a} \approx$
$4 \times 10^{-4} K^{-2}$, and $l \approx a$ at $25 \mathrm{~K}$. For case I, we have $\lambda \approx 2.0 a$, and for case II, we have $\lambda \approx 2.3 a$. Note however, that measurements of $I_{0}(T)$ at low temperature are difficult, and the error bars on $\mathcal{P}_{0}$ and $d \mathcal{P}_{0} / d E_{a}$ are significant. Therefore, the values given here are to be taken only as estimates of the order of magnitude.

Clearly, the echo decay is not consistent with slow spatial variations of the activation energy, but rather with disperse, localized point or line minima. A possible interpretation of the small spatial regions of small $E_{a}$ is that these regions constitute domain walls between large regions of coupled spins. In the large domains, the collective fluctuation rate $\left(\tau^{-1}\right)$ would be small, and $T_{1}$ very fast. Calculations of the echo decay for a linear trough of minima in $T_{1}$, rather than the point minima in cases I and II, are not consistent with the data. The reason is that the nuclei along the trough remain like nuclei, and the echo decay does not become exponential. Such a case is idealized; however one cannot rule out a situation in which the line minima fluctuates and neighbors along the trough decouple. Further experiments to probe $\mathcal{P}\left(E_{a}\right)$ and the echo decay with greater precision are likely to shed more light on the spatial dependence of the local activation energies, and their relationship to the stripe lattice. Also, a careful analysis of the temperature dependance of $l$ may allow one to distinguish between case I and II.

This work was performed at Los Alamos under the auspices of the US Department of Energy, and at the Aspen Center of Physics.

[1] J. M. Tranquada et al., Nature, 375, 561 (1995); J. M. Tranquada et al., Phys. Rev. B 54, 7489 (1996).

[2] J.M. Tranquada et al., Phys. Rev. B 59, 14712 (1999).

[3] M-H. Julien et al, Phys. Rev. Lett. 83, 604 (1999).

[4] N. J. Curro et al., Phys. Rev. Lett. 85, 642 (2000)

[5] J. Schmalian et al., Phys. Rev. Lett. 85, 836 (2000)

[6] C. P. Slichter, Principles of Magnetic Resonance, 3rd. Ed. (Springer Verlag, New York, 1990).

[7] B. Fine, cond-mat/9911229

[8] C. H. Pennington and C. P. Slichter, Phys. Rev. Lett. 66, 381 (1991)

[9] J. Bobroff et al., Phys. Rev. Lett. 79, 2117 (1997)

[10] S. Yu et al., Phys. Rev. Lett. 83, 3924 (1999)

[11] D. Thelen and D. Pines, Phys. Rev. B 49, 3528 (1994)

[12] R. E. Walstedt and S- W. Cheong, Phys. Rev. B 51, 3163 (1995)

[13] C. H. Recchia, K. Gorny and C. H. Pennington, Phys. Rev. B 54, 4207 (1996)

[14] N. J. Curro, C. P. Slichter, J. Mag. Res. 130, 186 (1998).

[15] N. J. Curro, T. Imai, C. P. Slichter, and B. Dabrowski, Phys. Rev. B 56, 877 (1997).

[16] A. Hunt et al., Phys. Rev. Lett. 82, 4300 (1999).

[17] N. J. Curro et al., Physica C 3411797 (2000) 
[18] J. Haase et al., Phys. Rev. B 59, 7191 (1999)

[19] A. J. Millis, H. Monien, and D. Pines, Phys. Rev. B 42, 167 (1990)

[20] V. Barzykin and D. Pines, Phys. Rev. B 52, 13585 (1995) 